\title{
BIM y CES. Dos agendas de gobierno unidas a través de las Tecnologías Digitales
}

\author{
BIM and CES. Two government agendas brought together through ICT tools
}

\author{
Danny Alfredo Lobos Calquín \\ Universidad de las Américas, Chile \\ dlobos@udla.cl \\ Lorena Del Pilar Silva Castillo \\ Pontificia Universidad Católica de Chile, Chile \\ Idsilva1@uc.cl
}

\begin{abstract}
The paper discusses the intersection of two main agendas for Chilean governments that are BIM (Building Information Modeling) and BEAM (Building Environmental Assessment Methods). It shows the state-of-the-art in both fields in Chile as well as in the world, it discusses some previous efforts of integration; it found some new opportunities for collaboration and finally proposes a new framework that brings together BIM and BEAM. The development of the method includes BIM Models, databases and spread sheets for building energy Certification, it finally provides a semi-automatic environment where architects model their design in BIM and this Information is used as an input to the certification process. Potentials and risk of this method are discussed.
\end{abstract}

Keywords: BIM (Building Information Modeling); BPS (Building Performance Simulation); BEAM (Building Environmental Assessment Methods); Architectural Design; Interoperability.

\section{Introducción}

En los últimos años, con la necesidad de construcción sustentable han incrementado rápidamente varios factores, como costos energéticos, el efecto de $\mathrm{CO} 2$ en la Tierra, tendencias públicas y gubernamentales, y la disponibilidad de nueva tecnología, ha llevado a requerir mejor desempeño en la edificación. Varios tipos de software BPS (Building Performance Simulation) han sido utilizados para simular demandas energéticas, iluminación, ganancias de calor, emisiones de $\mathrm{CO} 2$, etc. Muchos edificios están siendo certificados en estos aspectos utilizando Building Environmental Assessment Methods, o BEAM (Burnett \& Yik, 2001; IGBC, 2013), tales como LEED (US), BREEAM (UK), DGNB (Alemania), y CES (Chile), todos estos requieren una gran cantidad de compleja información del edificio para completar todos los documentos y formularios requeridos. Por otro lado, las tecnologías Building Information Modeling (BIM) apoyan todo el ciclo de vida de construcción y los modelos BIM poseen una gran cantidad geometría e información que tiene un gran potencial de apoyar fuertemente el análisis energético y evaluación de la energía de los edificios, incluso más útil para los equipos de proyectistas en etapas tempranas. Esta investigación apunta al intercambio automático de información entre estos tres entornos.

\section{Estado actual del arte en los tres campos}

A continuación, se discutirá el estado del arte de tres áreas: BIM, BPS y BEAM.

\section{BIM (Building Information Modeling)}

Las tecnologías BIM son el actual paradigma en práctica para Edificación e Infraestructura (NBS, 2016; Eastman et al., 2011; Jones, 2014). La adopción masiva comenzó en 2000 , y desde 2003 la implementación del gobierno comenzó como un estándar para las construcciones públicas con el programa BIM 3D-4D en GSA (GSA, 2016), Estrategia de construcción de gobierno en UK (Government Construction Strategy UK, 2016), Staatsbyg en Finlandia. Etapas tempranas de diseño son también plenamente apoyadas (Lobos, 2011).

En la actualidad casi todas las compañías de software BIM incluyen una herramienta de análisis energético en su paquete comercial BIM (ver Tabla 1). Por ejemplo Autodesk Revit posee una extensión llamada Insight 360 que ejecuta análisis de iluminación y validación de LEED v3 IEQc8.1 y LEED v4 IEQ Daylight Credit, Option 2 (Stine, 2015). La herramienta de simulación de Bentley AECOsim Energy Simulator Tool ejecuta simulaciones que generan documentación y reportes, los cuales son conformes a ASHRAE Standard 90.1 y certificación LEED (Bentley, 2016). Allplan Nemetscheck proporciona plantillas de software para obtener LEED Sustainable Site Credit 5.1 durante el diseño conceptual (Ich_BiM, 2016). Se puede concluir que los vendedores de BIM se han preocupado por la integración con LEED, la 
mayoría de ellos proponiendo el trabajo dentro de su entorno BIM, pero en última instancia no hay evidencia sólida de casos de pruebas, o proyectos, reales o su uso entre consultores energéticos.

\section{Situación de los software BPS (Building Performance Simulation)}

Los software BPS han demostrado su utilidad en la simulación de los aspectos de rendimiento del edificio (emisiones de CO2, Crédito LEED de Análisis-Iluminación, Cargas Térmicas, Solar, Acústico, etc.) con mayor precisión y velocidad. Algunos de los softwares más conocidos incluyen DesignBuilder, TAS, Daysim, Green Building Studio, y IES_VE, entre otros (Crawley et al. 2005; Attia et al., 2012; Clarke \& Hensen, 2015; Raslan \& Davies, 2010; Best Directory, 2016). Sin embargo, su usabilidad requiere una larga formación y profundo conocimiento de la energía del edificio, lo que complica la participación en tiempo real y continúa de arquitectos/diseñadores. Muchos de estos softwares pueden generar documentación LEED.

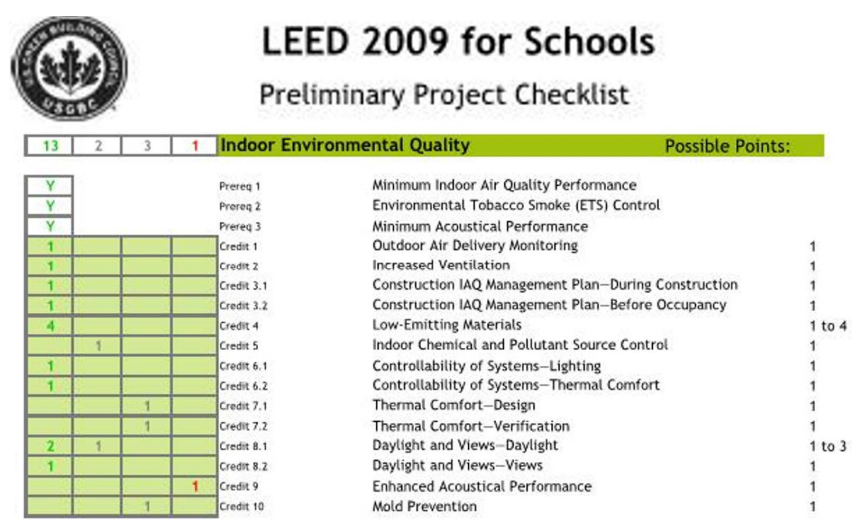

Figura 1: Tabla Checklist Certificación LEED. Fuente: https://www.usgbc.org/

A pesar de que el intercambio de información BIM-BPS ha sido propuesto por muchos autores (Bazjanac \& Maile, 2004; Calquin, Wandersleben, and Castillo, 2014; Hoffer, 2009; Prada-Hernandez, Rojas-Quintero, Vallejo-Borda \& PonzTienda, 2015) no hay pruebas sólidas de su uso mixto en la práctica

\section{Algunos esquemas BEAM (Building Environmental Assessment Methods).}

Los esquemas de evaluación tales como LEED (US), BREEAM (UK), DGNB (Alemania), y CES (Chile) y muchos otros, han estimulado al mercado inmobiliario y al gobierno proporcionando más confianza en la sustentabilidad de un edificio (Wu et al., 2016; Ramírez-Villegas et al., 2016; Seinre et al., 2014). Normalmente las planillas de estos sistemas se basan en créditos para cada criterio y reconocen diferentes tipos de construcciones, etapas, y categorías de certificación y llenan mirando los planos o copiando/pegando información de diversas fuentes. Sin embargo, no todos ellos están fuertemente conectados al software BIM o BPS, ya que muchos datos tienen que ser actualizados manualmente desde cualquier simulación o modelo 3D. Comúnmente todos los esfuerzos realizados para fabricar modelos BIM se pierden cuando los consultores de energía comienzan a evaluar el edificio para obtener la certificación BEAM (LEED, BREEAM, DGNB).

Tabla 1: Lista de herramientas de análisis de energía dentro de software BIM. Preparado por el autor, 2016.

\begin{tabular}{|c|c|c|c|c|c|}
\hline $\begin{array}{l}\text { PACK BIM y } \\
\text { compañía } \\
\text { vendedora }\end{array}$ & $\begin{array}{l}\text { Software de } \\
\text { análisis de } \\
\text { energía } \\
\text { incorporado/ } \\
\text { Extensión/ } \\
\text { Plugin / }\end{array}$ & 邑 & 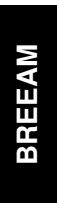 & $\frac{m}{z}$ & 㤩 \\
\hline \multirow[t]{2}{*}{$\begin{array}{l}\text { Revit } \\
\text { (Autodesk) }\end{array}$} & Insight 360 & $\begin{array}{l}\text { LEED } \quad \text { v3 } \\
\text { IEQc8.1 and } \\
\text { LEED v4 IEQ } \\
\text { lluminación } \\
\text { Natural, Option } \\
2\end{array}$ & -- & $* *$ & $\star *$ \\
\hline & $\begin{array}{l}\text { Light Analysis } \\
\text { Plugin }\end{array}$ & & & & \\
\hline $\begin{array}{l}\text { Archicad } \\
\text { (Graphisoft) }\end{array}$ & $\begin{array}{l}\text { EcoDesigner } \\
\text { Star }\end{array}$ & $\begin{array}{l}\text { ASHRAE 140- } \\
2007, \text { ASHRAE } \\
90.1-2007 \\
\text { (LEED Energía) }\end{array}$ & * & ** & $* *$ \\
\hline $\begin{array}{l}\text { AECOSim } \\
\text { (Bentley) }\end{array}$ & $\begin{array}{l}\text { AECOsim } \\
\text { Energy } \\
\text { Simulator }\end{array}$ & $\begin{array}{l}\text { ASHRAE } \\
\text { Standard } 90.1 \\
\text { Conformidad y } \\
\text { certificación } \\
\text { LEED. }\end{array}$ & -- & ** & $\star \star *$ \\
\hline $\begin{array}{l}\text { Digital } \\
\text { Project } \\
\text { (Gehry } \\
\text { Technologies } \\
\text { ) }\end{array}$ & -- & -- & -- & ** & -- \\
\hline $\begin{array}{l}\text { Allplan } \\
\text { (Nemetschec } \\
\text { k) }\end{array}$ & $\begin{array}{l}\text { Software } \\
\text { templates }\end{array}$ & $\begin{array}{l}\text { Plantilla puede } \\
\text { obtener } \\
\text { Sustainable } \\
\text { Site Credit } 5.1 \\
\text { (diseño } \\
\text { conceptual) }\end{array}$ & & ** & -- \\
\hline \multicolumn{6}{|c|}{$\begin{array}{l}{ }^{*} \text { A través de gbXML archivo a DesignBuilder. } \\
\text { ** Bajo investigación actual. Potencial usando IFC y modelos } \\
\text { BIM-basados en automatización del Chequeo. } \\
\text {-- No hay información/Evidencia }\end{array}$} \\
\hline
\end{tabular}

\section{Deficiencias detectadas}

Muchos problemas, tales como: la interoperabilidad BIM y BPS, los desafíos en el proceso de diseño integrado, la falta de estándares de interfaz y modelo, los requisitos de la evaluación del desempeño de edificios y los modelos de energía del edificio son actualmente de interés para los profesionales e investigadores. Las tres áreas (BIM, BPS y BEAM) no están bien conectadas. La información del software BIM y BPS normalmente no se comparte correctamente en el entorno BEAM o viceversa. Los primeros esfuerzos entre BIM y energía (Schlueter \& Thesseling, 2009) no incluyeron la integración de BEAM. Algunos esfuerzos recientes incluyen la integración de sistemas BIM y LEED en la etapa de diseño conceptual (Jalaei \& Jrade, 2015). Sin embargo, toda la información requerida en los documentos BEAM debe ser completada manualmente (USGBC, 2016) (CES, 2016b) y la nueva información debe ser actualizada manualmente 
después de los cambios de diseño (Kryegel \& Nies, 2008) tales como añadir/borrar pisos/áreas/recintos, cambiar el tamaño/rotar recintos, mover muros, cambio de material, etc. Proporcionar información de rendimiento requerida/lograda a los "equipos de proyectistas". Es también una deficiencia detectada en estos paquetes de software.

\section{Integraciones propuestas previamente}

Informes recientes de investigación de mercado revelan el gran potencial de integración para la industria (Jones, 2014). Bazjanac (2008) fue uno de los primeros autores que se ocuparon del intercambio BIM-IFC para mejorar el diseño energético de los edificios. También han habido algunos esfuerzos recientes en relación con la integración de estos tres campos, como la integración de BIM y el sistema LEED en la etapa de diseño conceptual (Jalaei y Jrade, 2015), pero dependiendo de un paquete BIM comercial (Autodesk Revit); utilizando cloud-BIM para la automatización LEED (Wu \& Issa, 2013); plan de ejecución BIM en proyectos de edificios sustentables (Wu \& Issa, 2015); ciertas pautas para el uso de BIM en el análisis energético de edificios (Reves, Svetlana, \& Issa, 2015); y mapeo de procesos integrados para la implementación de BIM en la entrega de proyectos de edificios sustentables (Wu \& Issa, 2013). Remmen et al. (2015) promueve un framework abierto para la simulación de desempeño de edificios basado en BIM utilizando Software Modelica. A principios de los años 2000, Bayforrest trató de conectar un modelo de producto compatible con el modelo IFC de un edificio (utilizando Autodesk Architectural Desktop, un software proto BIM) a través de Internet con bases de datos para los recursos y requerimientos energéticos de materiales de construcción (Neuberg et al, 2004) pero no conectado a ningún esquema BEAM. Se ha propuesto el uso de chequeo de modelos basado en BIM BMC (BIM model checking) para la versión danesa del DGNB (Gade, Svidt y Jensen, 2016).

En conclusión, no hay un método directo para llenar los formularios de BEAM directamente del software BIM con el fin de acelerar y hacer que el proceso de certificación de un edificio sea más preciso. Además, hay una falta de una interfaz intuitiva para hacer estos procesos.

\section{Método aplicado en esta investigación}

Se ha propuesto un nuevo framework (Figura 2), incluyendo el intercambio de información entre BIM y BEAM. En este framework se comienza reconociendo los requisitos de BEAM (es decir, LEED, BREEAM, DGNB, CES de Chile), modelamos luego el BIM para el diseño arquitectónico (bajo protocolos BPS), luego se simula el BIM en un entorno BPS (Design Builder, TAS, Ecodesigner, Green Building Studio, etc.), Io cual arrojara como resultado hacer cambios, se realizan cambios en el modelo BIM y se cumplen así los requisitos de BEAM y el edificio pueda certificarse.
Tabla 2: Criterios CES que se pueden obtener desde la herramienta BIM. Elaborado por los autores, 2016

\begin{tabular}{|c|c|}
\hline Categoría & Variable \\
\hline \multirow{5}{*}{$\begin{array}{l}\text { Información } \\
\text { General }\end{array}$} & Ubicación \\
\hline & Propietario \\
\hline & Nombre del Proyecto \\
\hline & ID cliente \\
\hline & Superficie \\
\hline \multirow{2}{*}{ Uso y Localización } & Ciudad de Emplazamiento \\
\hline & Días de uso/semanas \\
\hline \multirow{8}{*}{$\begin{array}{l}\text { Definición de } \\
\text { habitaciones }\end{array}$} & Numeración de recintos \\
\hline & Nombre habitación \\
\hline & Descripción \\
\hline & Área útil \\
\hline & Densidad de uso \\
\hline & Carga de iluminación \\
\hline & Cargas de Equipos \\
\hline & ¿Regularmente Ocupada? \\
\hline \multirow{2}{*}{$\begin{array}{l}\text { Definición de } \\
\text { Grupos }\end{array}$} & Numeración de Grupos \\
\hline & Nombres de Grupos \\
\hline \multirow{3}{*}{$\begin{array}{c}\text { Asignación de } \\
\text { Recintos a los } \\
\text { distintos Grupos }\end{array}$} & Numeración de recintos \\
\hline & Nombre de reciento \\
\hline & Grupo al que pertenece \\
\hline \multirow{4}{*}{ Envolvente } & Área / Altura \\
\hline & Material (Paredes / Techos / Piso) \\
\hline & Espesor (Paredes / Techos / Piso) \\
\hline & Aislación (Paredes / Techos / Pisos) \\
\hline \multirow{2}{*}{$\begin{array}{c}\text { Infiltración y } \\
\text { renovación de aire }\end{array}$} & Infiltraciones y renovaciones aire \\
\hline & Ventilación Nocturna \\
\hline \multirow{4}{*}{ Radiación } & Orientación \\
\hline & Dimensión \\
\hline & Tramitancia Luz visible \\
\hline & Factor Solar Vidrio \\
\hline $\begin{array}{l}\text { Características de } \\
\text { los marcos Vanos } \\
\text { por orientación }\end{array}$ & $\begin{array}{l}\text { Dimensiones del obstáculo de la } \\
\text { fachada }\end{array}$ \\
\hline \multirow{4}{*}{ Ventilación } & Área recinto \\
\hline & Altura \\
\hline & Uso \\
\hline & Densidad Ocupacional \\
\hline
\end{tabular}

A continuación, se presenta y discute un caso para este framework. Se desarrolló un método semi-automatizado de intercambio de información entre una plataforma BIM (Autodesk Revit) y la certificación chilena (CES). El framework propuesto se basó en el conocimiento de ambos temas (BIM y BEAM), que normalmente se separan y son desarrollados por profesionales. El método fue para mapear ambos procesos (BIM / BEAM), aquí se encontró una fuerte concordancia entre varias variables (Ver Tabla 2), el listado acá muestra aquellas que son requeridas por el CES y a la vez pueden ser extraídas desde el modelo BIM de Arquitectura. A continuación, se configuró una conexión de base de datos (MS 
Access) entre el software BIM propietario (Autodesk Revit 2017) y un formato de hoja de cálculo BEAM (parte del sistema de evaluación CES chileno), que permite completar y actualizar automáticamente la planilla desde el modelo BIM.

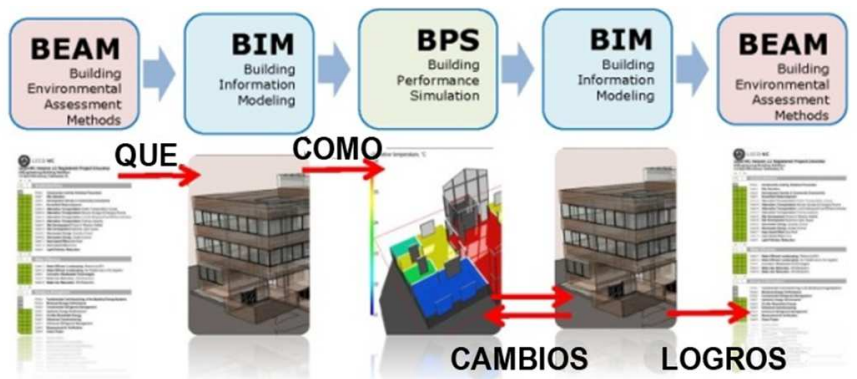

Figura 2: El nuevo framework propuesto. Fuente: autores (2016).

\section{Resultados de la investigación}

El resultado fue un nuevo flujo de información de BIM a BEAM. Una vez finalizada la configuración inicial (nombres, rutas y ubicaciones de los archivos), todos los cambios realizados en el modelo BIM se transmiten a la hoja de cálculo BEAM a través del vínculo de base de datos. Esto significa que después de cada cambio de diseño (piso, habitaciones, tamaños y nombres) realizado en el modelo BIM, toda la información requerida se transmite automáticamente a la hoja de cálculo BEAM sin embargo la frecuencia de actualizaciones finalmente debe hacerse manualmente. Una lista de los criterios tomados de BIM a BEAM se puede encontrar en la Tabla 2.

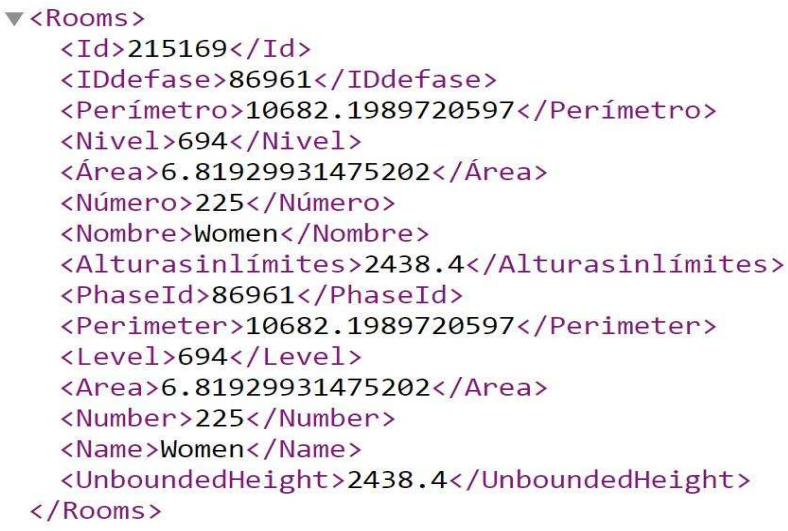

Figura 3: Información de habitación de BIM convertida en información XML. Preparado por los autores, 2016

La mayoría de los datos BIM se convierten en esquema XML (Figura 3), y luego reconocidos como información tabulada, y traducida al formulario de hoja de cálculo de CES. Esto permite compartir enormes cantidades de información de BIM a BEAM y también permite la independencia de cualquier software BIM específico, ya que la mayoría de ellos son capaces de exportar este tipo de archivos.

\section{Valoración de la contribución}

Actualmente, los documentos BEAM deben completarse manualmente. Los modelos BIM recogen una gran cantidad de información útil para los documentos BEAM y sólo unas pocas investigaciones tratan este problema y lo abordan parcialmente. El flujo de trabajo propuesto crea una nueva conexión automatizada entre la información BIM y los documentos BEAM. Proporcionar la información de desempeño requerida/lograda a los equipos de proyectista es también una contribución significativa de esta investigación. Este caso muestra el uso del framework propuesto en un flujo de trabajo específico (Revit-CES), podría aplicarse a Archicad-LEED, Bentley-BREEAM o cualquier otra combinación.

\section{Conclusiones}

La investigación muestra que una gran cantidad de información útil agregada a los modelos BIM puede ser reutilizada en los esquemas BEAM. Actualmente no existe una integración real entre los campos BIM-BPS-BEAM, este framework promueve dicha integración y podría apoyar el trabajo de las oficinas de arquitectura, de los consultores de eficiencia energética y de los organismos de estado que la promueven. Los mandatos BIM del gobierno normalmente no incluyen especificaciones BEAM claras, ni tampoco aplicaciones para vincularlas. Una excepción es el "GSA 3d$4 d$ program BIM" que incluye directrices claras para apoyar la modelación energética del edificio y la simulación desde modelos BIM. Los mandatos BIM y los esquemas BEAM de los gobiernos deben trabajar juntos más claramente, por ejemplo en Chile no existe un diálogo (a la fecha 2017) entre Plan BIM de CORFO o BIM Forum y los promotores del CES. Los promotores de CES han apoyado desde un inicio los intentos de integración con BIM. Los esquemas regionales de evaluación como el CES chileno no son aplicables en otras regiones (Norteamérica, Asia, Europa, etc.), pero el método de intercambio es válido para otros sistemas (LEED, BREEAM, DGNB, etc.) en el mundo.

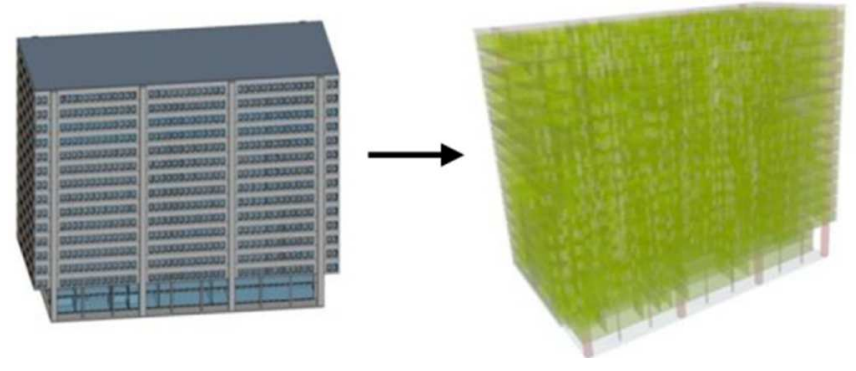

Figura 4. Ejemplo de guías de energía de GSA: un análisis de energía basado en BIM que comienza con un modelo BIM exportado a un comprobador de modelos. Fuente: GSA (2016).

\section{Mirada Futura}

Algunos aspectos deben ser abordados en futuros trabajos, tales como: casos de estudio, integración web, cumplimiento de políticas energéticas integradas de gobierno, facilidad de 
uso, una interfaz más completa con otros programas BIM (Archicad, Bentley, Allplan, Digital Project), intercambio de información desde/hacia BPS (Design Builder, IES_VE, TAS, etc) puede ser abordada. Algunos problemas potenciales como el retraso operacional y el cruzamiento de información deben ser discutidos como un riesgo para la implementación de este tipo de framework en las oficinas de arquitectura y consultoría energética. Debe además reglarse el cumplimiento de IFC para promover estándares de OpenBIM, y finalmente una vinculación más directa a los motorres Energy+ y DOE2.

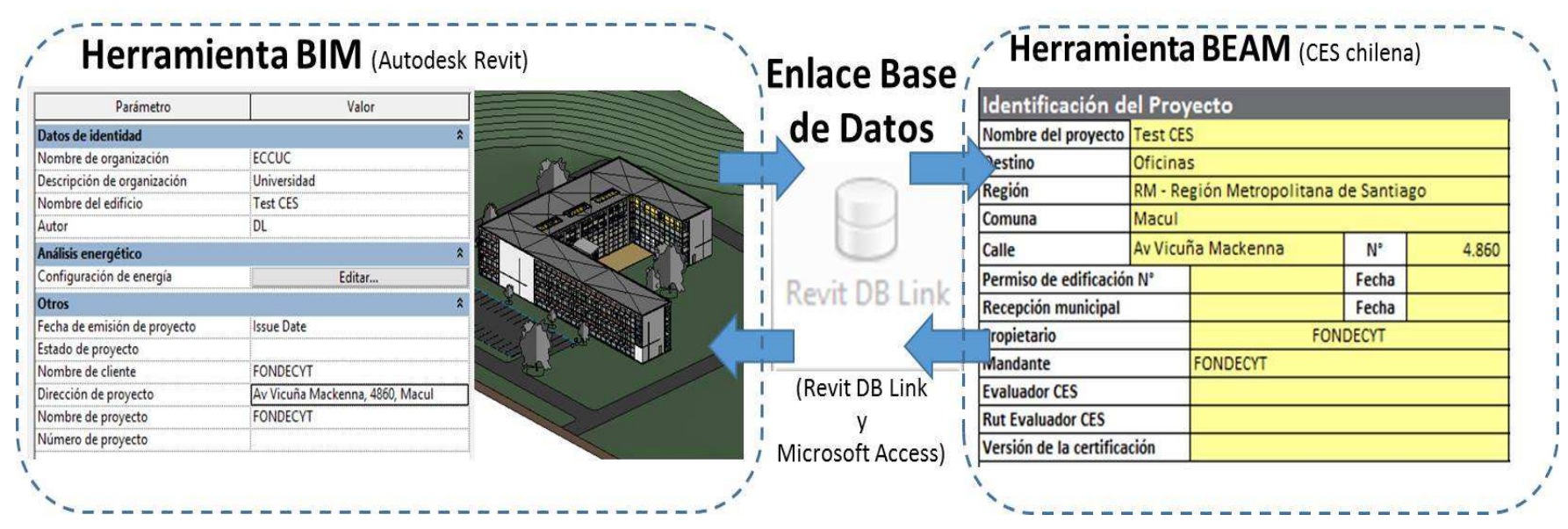

Figura 5: El modelo BIM y los datos básicos transferidos a la herramienta BEAM. Preparado por los autores, 2016

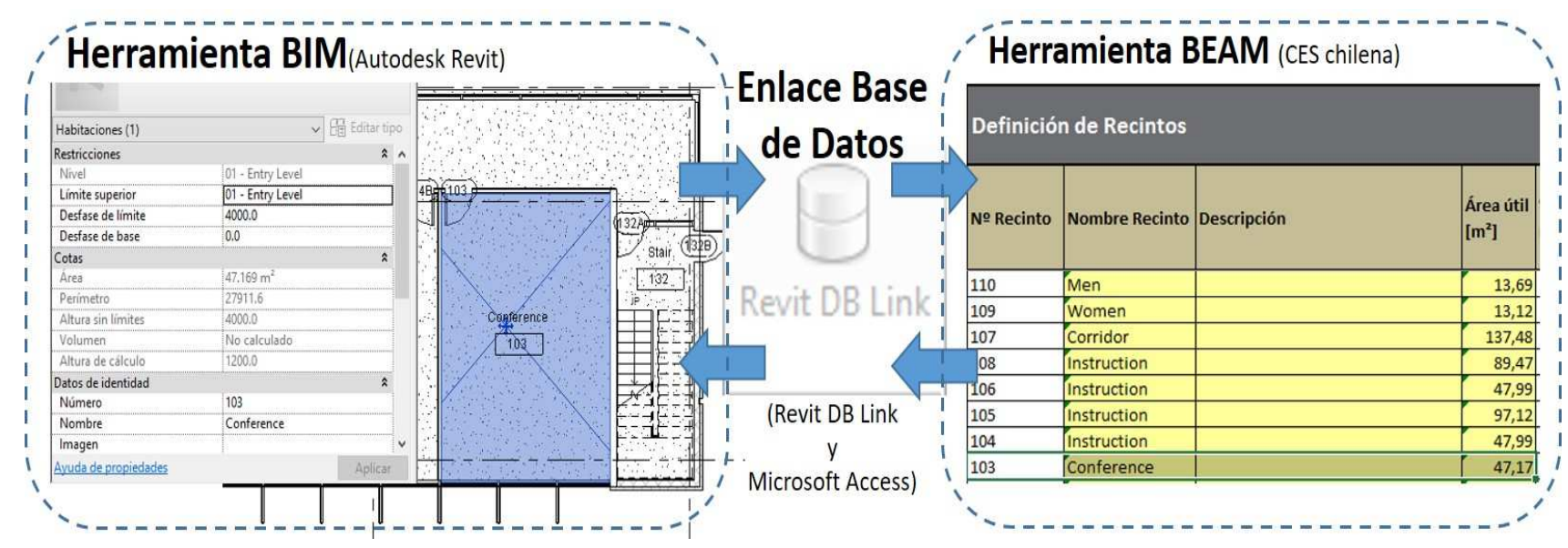

Figura 6: cambios en el modelo BIM (tamaño de la habitación y actualización del nombre) transferidos a la herramienta BEAM. Preparado por los autores, 2016

\section{Agradecimientos}

Esta investigación ha sido financiada por la Comisión Nacional de Investigación Científica y Tecnológica Chilena (CONICYT) a través de FONDECYT 11151024, también es apoyada por el Ministerio chileno de Obras Públicas (MOP-DA, Ministerio de Obras Públicas, Dirección de Arquitectura), Chile GCB (Green Building Council), Consultora BIM-Chile, CITEC UBB, Consultora EA-Buildings e Instituto de la Construcción.

\section{Referencias}

Attia, S., Hensen, J. L. M., Beltrán, L., \& De Herde, A. (2012). Selection criteria for building performance simulation tools: contrasting architect's and engineer's needs. Journal of Building Performance Simulation, 5(3), 155-169. doi:10.1080/19401493.2010.549573

Bazjanac, V. (2008). IFC BIM-Based Methodology for SemiAutomated Building Energy Performance Simulation. CIB-W78 25th Conference, July 15-17, 2008, Santiago, Chile

Bazjanac, V. and Maile, T. (2004). IFC HVAC interface to EnergyPlus - A case of expanded interoperability for energy simulation. SimBuild 2004. Boulder (Colorado, US)

Bentley Institute. (2016). AECOsim Energy Simulator V8i Fundamentals (imperial). Bentley Systems, Incorporated. 
BEST Directory. (2016). Building Energy Software Tools. Retrieved 15, December, 2016, from http://www.buildingenergysoftwaretools.co m

Burnett, J., \& Yik, F. W. (2001). Framework of Building Environmental Assessment Methods. HKIE Transactions, 8(3), 1-7. ISSN: 1023$697 X$

Calquin, D. A. L., Wandersleben, G., \& Castillo, L. S. (2014). Interoperability map between BIM and BPS software. Proceedings of the 2014 International Conference on Computing in Civil and Building Engineering.

CES (2016a). Certificación Edificio Sustentable. Retrieved December 15, 2016, from http://www.certificacio nsustentable.cl/

CES (2016b). Manual de Herrmientas de Evaluación y Calculo. Retrieved December 15, 2016, from http://www.certificacio nsustentable.cl/

Clarke, J. A., \& Hensen, J. L. M. (2015). Integrated building performance simulation: Progress, prospects and requirements. Building and Environment, 91, 294-306. doi:10.1016/j.buildenv.2015.04.002

Crawley, D. B., Hand, J. W., Michaël, K., \& Griffith, B.T. (2005). Contrasting the Capabilities of Building Energy Performance Simulation Programs. Ninth International IBPSA Conference, Montréal, Canada.

Eastman, C., Paul, T., Rafael, S., \& Kathleen, L. (2011). BIM Handbook: A Guide to Building Information Modeling for Owners, Managers, Designers, Engineers and Contractors (2nd ed.): John Wiley \& Sons.

Gade, P., Svidt, K., \& Jensen, R. (2016). Analysis of DGNB-DK criteria for BIM-based Model Checking automatization. Retrieved from http://vbn.aau.dk/da/publications/analysis-of-dgnbdk-criteria-forbimbased-model-checking-automatization\%28df19474a-059443ad-b41f-5785d2155da8\%29.html

Government Construction Strategy UK (2016). Government Construction Strategy: 2016-2020. Retrieved from https://www.gov.uk/government/publications/governmentconstruction-strategy-2016-2020

GSA. (2016). GSA 3D-4D BIM Program. Retrieved from https://www.gsa.gov/portal/category/21062

Hoffer, E. R. (2009). BIM for LEED. Retrieved from http://images.autodesk.com/adsk/files/bim_for_leed_0509.pdf

Ich BiM. (2014). Ich BiM to Leed to Allplan. [Video File]. Retrieved from https://www.youtube.com/watch?v=fe7Tplh 4V40

IGBC. (2013). Building Environmental Assessment Method for Ireland. Irish Green Building Council.

Jalaei, F., \& Jrade, A. (2015). Integrating building information modeling (BIM) and LEED system at the conceptual design stage of sustainable buildings. Sustainable Cities and Society, 18, 95-107. doi:10.1016/j.scs.2015.06.007

Jones, S. (2014). Recent SmartMarket BIM Research by McGraw Hill Construction. McGraw Hill Construction.

Kryegel, E. \& Nies, B. (2008) Green BIM. Wiley: Indianapolis.

Lobos, D. (2011). BIM Supported Building Envelopes and Space Layout Based on a Case Study in South America. Weimar: Bauhaus-Universität. ISBN-10: 3957730376.

NBS. (2016). National BIM Report 2016. Retrieved from https://www.thenbs.com/-/media/files/pdf/bim-report2016.pdf?la=en

Neuberg, F., Fank, E., \& Ekkerlein, C. (2004). Integrated Life Cycle Simulation and Assessment of Buildings. International Conference on Computing in Civil and Building Engineering, ICCCBE, Weimar.
Prada-Hernandez, A., Rojas-Quintero, J. S., Vallejo-Borda, J., \& PonzTienda, J. L. (2015). Interoperability of Building Energy Modeling (BEM) with Building Information Modeling (BIM). SIBRAGEC ELAGEC 2015, San Carlos.

Ramírez-Villegas, R., Eriksson, O., \& Olofsson, T. (2016). Assessment of renovation measures for a dwelling area - Impacts on energy efficiency and building certification. Building and Environment, 97, 26-33. doi:10.1016/j.buildenv.2015.12.012

Raslan, R., \& Davies, M. (2010). Results variability in accredited building energy performance compliance demonstration software in the UK: an inter-model comparative study. Journal of Building Performance Simulation, 3(1), 63-85. doi:10.1080/19401490903477386

Reeves, T., Olbina, S., \& Issa, R. (2015). Guidelines for Using Building Information Modeling for Energy Analysis of Buildings. Buildings, 5(4), 1361-1388. doi:10.3390/buildings5041361

Remmen, P., Cao, J., Ebertshäuser, S., Frisch, J., Lauster, M., Maile, T., Wimmer, R. (2015). An open framework for integrated BIMbased building performance simulation using Modelica. Proceedings of 14th IBPSA, Hyderabad.

Schlueter, A., \& Thesseling, F. (2009). Building information model based energy/exergy performance assessment in early design stages. Automation in Construction, 18(2), 153-163. doi:10.1016/j.autcon.2008.07.003

Seinre, E., Kurnitski, J., \& Voll, H. (2014). Building sustainability objective assessment in Estonian context and a comparative evaluation with LEED and BREEAM. Building and Environment, 82, 110-120. doi:10.1016/j.buildenv.2014.08.005

Stine, D. (2015). Building Performance Analysis in Revit 2016 R2 with Autodesk Insight 360. AECbytes Tips and Tricks (Issue \# 76).

USGBC. (2016). Guide to LEED Certification - Commercial. (8). Retrieved 15, December, 2016, from http://www.usgbc.org/certguide

Wu, P., Mao, C., Wang, J., Song, Y., \& Wang, X. (2016). A decade review of the credits obtained by LEED v2.2 certified green building projects. Building and Environment, 102(June 2016), 167178. doi:10.1016/j.buildenv.2016.03.026

Wu, W., \& Issa, R. (2010). Feasibility of integrating building information modeling and LEED certification process. Proceedings of the International Conference on Computing in Civil and Building Engineering, Nottingham.

Wu, W., \& Issa, R. (2015). BIM Execution Planning in Green Building Projects: LEED as a Use Case. American Society of Civil Engineers, 31(1), doi:10.1061/(ASCE)ME.1943-5479.0000314 